\title{
Three-dimensional image sensing by chromatic confocal microscopy
}

\author{
H. J. Tiziani and H.-M. Uhde
}

\begin{abstract}
In the image of a confocal microscope, only those parts of an object appear bright that are located in the focal plane of the objective. Because of an axial chromatic aberration deliberately introduced into the microscope objective, the location of the focal plane depends on the wavelength used. By using a white-light source and examining an object with a depth variation less than the axial range of the chromatic focus, we find that all parts of the object appear sharp and bright in the image, but according to its height they appear in different colors. A camera with black-and-white film sequentially combines, with three selected chromatic filters, intensity and tone of color of each object point. For each tone of color one can assign a height by using a calibration curve. This assignment could be made unequivocal by the selection of filters with adequate chromatic transmission.
\end{abstract}

Key words: Three-dimensional analysis, confocal microscopy, chromatic measuring.

\section{Introduction}

For the three-dimensional analysis of structures in the macroscopic as well as in the microscopic range, the confocal principle is suitable, leading to a true depth discrimination. ${ }^{1-3}$ By image formation in the image plane, parts of the object located inside the object plane appear sharp and bright, whereas parts outside the object plane appear blurred and dark. For objects with no intensity variations but only height structures, the three-dimensional structure can be analyzed. Other methods, developed by Agard and Sedat ${ }^{4}$ and by Erhardt et al., ${ }^{5}$ record a series of images from the microscope with different focus settings. The blurred sections are eliminated arithmatically. For the remaining sharp reproduced parts of the object in each image, the focus shift of the relevant image gives the $z$ coordinate (along the optical axis). However, these methods work only if the objects have structures with different reflectivities to distinguish between sharp or blurred structures. Before introducing the chromatic method in Section 3, we describe in Section 2 a confocal setup that uses a rotating Nipkow disk.

The authors are with the Institute für Technische Optik der Universität Stuttgart, Pffaffenwaldring 9, D-7000 Stuttgart 80, Germany.

Received 8 October 1992.

0003-6935/94/101838-06\$06.00/0.

(1) 1994 Optical Society of America.

\section{Confocal Microscopy Using a Rotating Nipkow Disk}

In confocal microscopy ${ }^{6-9}$ a point light source is reproduced by an objective at point $\mathrm{P}$ on the object (Fig. 1). The light spot at $\mathrm{P}$ is collected by an objective and is focused onto a point detector by a pinhole in front of the detector. In this constellation the detector signal has its maximum. If the object is shifted axially, the incoming light is distributed onto an object area around P. The brightness on the object at $\mathrm{P}$ is reduced. Furthermore, this light is no longer focused onto the point detector. A great portion of the light is obstructed by the pinhole in front of the detector. Therefore shifting the object axially strongly reduces the detector signal.

By observing the intensity at the detector, we can determine the $z$ coordinate of $\mathrm{P}$ with high accuracy. To analyze the whole object, we must scan the light spot across the object area. As an alternative to object scanning, a rotating Nipkow disk (or a disk with a pinhole) can be used as shown in Fig. 2, where such an arrangement is shown. Several points on the object are now illuminated simultaneously. To work in reflection, for instance, the light spots should be diffraction limited, and the distance between the holes on the disk must be chosen appropriately to prevent an overlap of the spot images. No light passing one hole should return through a neighboring hole. Otherwise the depth discrimination would be reduced. The detector can be replaced by an eyepiece or a CCD camera. If the Nipkow disk is rotating fast enough, a complete picture of the object can be observed in which only those parts of the object 


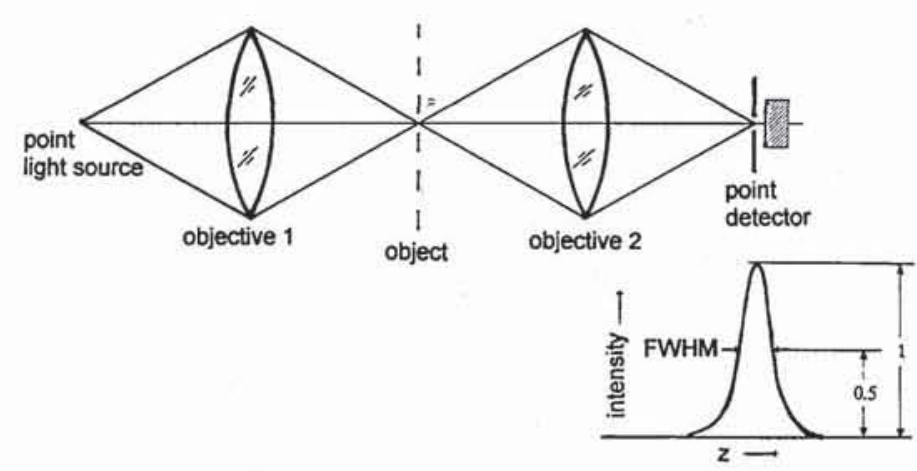

Fig. 1. Depth discrimination with confocal reproduction.

appear bright that are located in the common focus of the objectives (Fig. 2). This leads to a height selection of the object. In reflection only one objective and one Nipkow disk are necessary.

For the analysis of a three-dimensional object, the object is moved stepwise in an axial direction. At each position an image is stored in the frame grabber. One obtains, for instance, a series of 256 height sections. In each pixel the height section is searched for in which the pixel appears brightest. For the whole image we obtain a data set that can be interpreted to be an 8-bit-deep image. This image can be shown on the monitor. The intensity at each pixel gives the height of the object point belonging to this pixel. To obtain additional information about the reflectivity of each object point, we synthesize an autofocus picture out of the 256 height sections. In the autofocus image each pixel gets the maximum

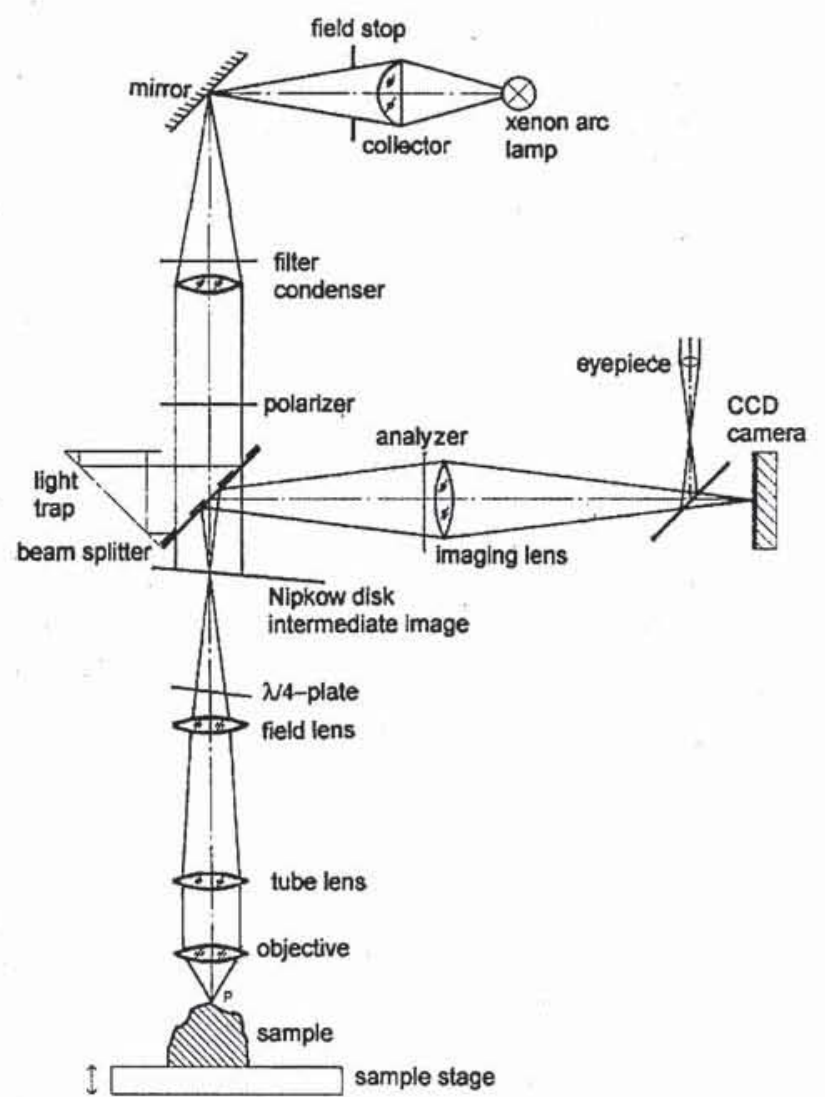

Fig. 2. Experimental setup of the scanning confocal microscope with the Nipkow disk. of its intensity values during the series of height sections. The result can be presented in many ways.

For example, the information of the autofocus image and the height image can be combined to generate stereoscopic images or anaglyphic images that can be viewed with red-green glasses. A better height determination is possible by taking the center of gravity of the intensity series instead of the maximum. It has been shown ${ }^{1,8}$ that, for the pseudoparaxial region, the intensity in the $z$ direction can be written approximately as

$$
I(z)=\left\{\frac{\sin [k z(1-\cos \alpha)]}{k z(1-\cos \alpha)}\right\}^{2},
$$

where $k$ is the wave number, $k=2 \pi / \lambda, \lambda$ is the wavelength, and $\sin (\alpha)$ is the numerical aperture of the image-forming system. The numerical aperture of the condenser optics is assumed to be the same.

In Eq. (1) the intensity drops to its half-maximum, i.e., to the 3-dB points (Fig. 1). For the FWHM we find

$$
\mathrm{FWHM}=\frac{0.44 \lambda}{1-\cos \alpha} .
$$

Furthermore, the point-spread function is the square of the point-spread function of a standard imaging microscopic system using the same objective. The transverse resolution in the region of $\Delta z$ is approximately 0.7 of the transverse resolution of a standard microscope. The FWHM has also been measured for the objective used mainly in our experiments, namely, the $160 \times / 0.95$ objective with a magnification of 160 and a numerical aperture of 0.95 .

There is some discrepancy between theoretical and practical results. The objective was especially chosen to have a longitudinal chromatic aberration of 5.1 $\mu \mathrm{m}$. Furthermore, a small centering error seems to be present. The field curvature of the objective with a long working distance was measured. The data of the $160 \times / 0.95$ objective are summarized in Table 1 . In addition, the experimentally obtained meansquare deviation $h_{q}$ and the mean deviation $h_{a}$ of the measured topographic heights $h$ are given. It should be pointed out that the FWHM in Table 1 was measured with a small spectral bandwidth of $\Delta \lambda=$ $21.7 \mathrm{~nm}$; the spectral bandwidth in Fig. 8 below was much larger. A white-light source and broadband

Table 1. Confocal Microscope Data Obtained by Using the $160 \times / 0.95$

\begin{tabular}{|c|c|}
\hline Objective & $160 \times / 0.95$ \\
\hline Image field $(\mu \mathrm{m})$ & $70 \times 50$ \\
\hline Field curvature $(\mu \mathrm{m})$ & 0.108 \\
\hline Theoretical FWHM ${ }^{2}(\mu \mathrm{m})$ & 0.352 \\
\hline Measured FWHM ${ }^{a, b}(\mu \mathrm{m})$ & 0.427 \\
\hline Mean-square height resolution $h_{q}(\mathrm{~nm})$ & 2.7 \\
\hline Mean height resolution $h_{a}(\mathrm{~nm})$ & 2.2 \\
\hline
\end{tabular}
Objective

${ }^{a} \lambda=545.7 \mathrm{~nm}$.

${ }^{b} \Delta \lambda=21.7 \mathrm{~nm}$. 
color filter were used, leading to an enlargement of the FWHM by a factor of 2 .

For the following practical applications the system was calibrated by using a perfect reference surface to compensate for systematic errors. For the calibration and compensation of systematic errors such as field curvature, a plane mirror with a surface quality better than $\lambda / 10$ is used as a reference. Figure 3 shows a measured topography of a microchip (field size of $70 \mu \mathrm{m} \times 50 \mu \mathrm{m}$ ), in which a resolution over the field of $6 \mathrm{~nm}$ was obtained after correcting the aberrations and defects of the detector elements.

\section{Chromatic Method}

Here we describe a chromatic method for confocal measurements. It is based on the depth registration by color coding, in which the chromatic length aberration of the objective is used. The objective should be perfectly corrected for the geometrical aberrations with respect to the different chromatic image planes. A compromise was found for the specially selected objectives.

In Ref. 6 a real-time confocal scanning optical microscope is indicated in which the specimen's stationary scanning illumination is generated by a broadband light source shining on a rotating mask with 200,000 pinholes. Each illuminating pinhole is also its own analyzing aperture. Using white light, Xiao et al. pointed out that chromatic aberrations of the objective lens ensure that images from different heights in the specimen are all present and all in focus but have different colors. The colors are apparent, although not striking, in the photographs of Ref. 6 . It is mentioned that if the illuminating light is put through a narrow-bandpass filter, height is revealed by intensity changes.

In our research specially selected objectives with chromatic length aberrations were used. It was

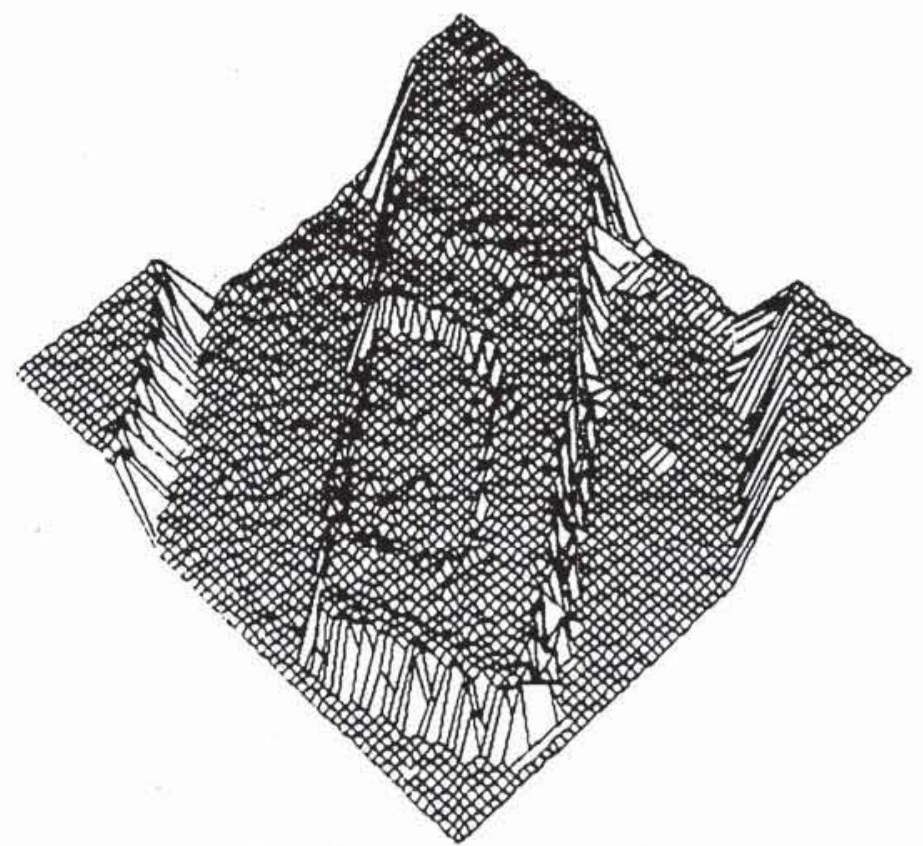

Fig. 3. Topography of a microchip calculated out of 256 hoight sections ( $z$ scanning, center of gravity calculation). found that, when using three colors, we obtained an unambiguous determination of the topography. It should be noted that for the depth discrimination the color impression was used. For one color only at least a two-height ambiguity occurs. Experiments using two colors only led to ambiguity problems in the overlapping region. Therefore, for unambiguous topography detection (Fig. 9 below) three colors are needed, leading in addition to a higher resolution. It should be stressed once more that color impression is selected for depth discrimination and not intensity only.

For investigations of the chromatic method, a specially selected $160 \times / 0.95$ objective with an axial chromatic aberration of $5.1 \mu \mathrm{m}$ and a corrected spherical aberration for the planes in question was used. The spectral sensitivity range of the system with the special objective with a long working distance was from 350 to $920 \mathrm{~nm}$.

In a confocal microscope only the object parts in focus appear bright. Therefore for an object to be examined with a depth variation less than $5.1 \mu \mathrm{m}$, all parts of the object appear simultaneously sharp and bright, but in different colors depending on their heights. Care had to be taken because the chromatic aberration was not linear with respect to wavelength variation. Viewing the image with an eyepiece, we observed a subjective impression of the threedimensional structure of the surface by the color appearance, without shifting the object axially. Through the use of white light, it is no longer necessary to take a series of height sections. All the three-dimensional information about the object is obtained on line. Recording the image with blackand-white film, one discards the height information with the color, but the resulting black-and-white image is an on-line autofocus image using color discrimination. It is no longer necessary to combine the autofocus image synthetically out of 256 height sections. This is a great advantage when no axial shift of the object is needed, as well as with respect to the time consumed for measurements. However, a small reduction in depth resolution occurred.

To determine the height information of one single image, we must analyze the image as a color image, in which a height is assigned to each pixel. One promising method of obtaining the height information by color coding is found by spectral analysis. It should be noted, however, that the color description is not a physical quantity but rather a sensory impression, which arises in the eye and the brain. This sensory impression results from light reaching the retina of the eye. Only the spectral intensity distribution $I(\lambda)$ of that incoming light is physically describable.

To determine the complete spectral intensity distribution of each object point is demanding. Therefore it was necessary to examine the essential components of the complete spectral distribution. This becomes possible by regarding the way the human eye works. On the retina are rods and cones. These send a signal to the visual center of the brain, whose inten- 
sity increases with increasing intensity of incoming light. The rods are very sensitive and permit viewing even in dark surroundings. The signal that they send to the brain can vary only in its intensity. It describes the brightness of the viewed scene. A further description of the light, i.e., its color is not possible. In darkness one can see only gray values. The cones are active only in bright light. Three kinds of cone exist with different spectral sensitivities (Fig. 4). These sensitivities are $E_{1}(\lambda), E_{2}(\lambda)$, and $E_{3}(\lambda)$ and have as light the spectral distribution $I(\lambda)$; we obtain signals of the three kinds of cone with the intensities $S_{1}, S_{2}$, and $S_{3}$ :

$$
\begin{aligned}
& S_{1}=k \int I(\lambda) E_{1}(\lambda) \mathrm{d} \lambda, \\
& S_{2}=k \int I(\lambda) E_{2}(\lambda) \mathrm{d} \lambda, \\
& S_{3}=k \int I(\lambda) E_{3}(\lambda) \mathrm{d} \lambda .
\end{aligned}
$$

The triple $\left(S_{1}, S_{2}, S_{3}\right)$ is what the brain interprets as color; hence color can be described as a threedimensional quantity, where $k$ is a constant factor. The complete description of light, which is given by $I(\lambda)$, is reduced to the three values $S_{1}, S_{2}$, and $S_{3}$. The triple $\left(S_{1}, S_{2}, S_{3}\right)$ can be transformed into

$$
\begin{aligned}
I & =S_{1}+S_{2}+S_{3}, \\
\left(X_{f}, Y_{f}\right) & =\left(\frac{S_{1}}{S_{1}+S_{2}+S_{3}}, \frac{S_{2}}{S_{1}+S_{2}+S_{3}}\right) .
\end{aligned}
$$

Then $I$ is the intensity of the light and $X_{f}$ and $Y_{f}$ are

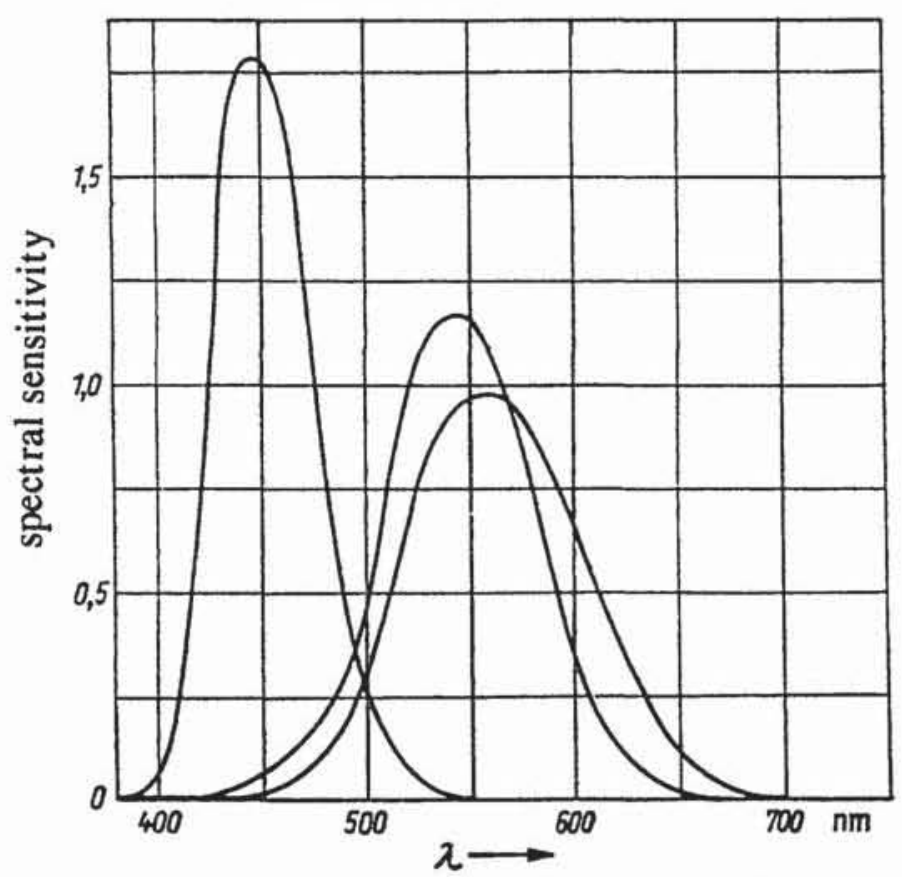

Fig. 4. Spectral sensitivity of the three kinds of cone of the human retina. ${ }^{9}$ two parameters, describing the tone of color, with $0 \leq X_{f} \leq 1$ and $0 \leq Y_{f} \leq 1$. The tone of color is a two-dimensional quantity. It does not change if all values of the triple $\left(S_{1}, S_{2}, S_{3}\right)$ change by the same factor, i.e., if the intensity changes.

One can stimulate the color sensitivity and the information transfer of the eye by taking an image with a black-and-white film several times in series and by placing a different chromatic filter in front of the camera or in front of the light source each time. The combination of camera and different filters corresponds to the different kinds of cones. The spectral transmission in commercial color film turned out to be unsuitable for the chromatic method. Chromatic filters with ideal transmissions were selected after some theoretical investigations and some practical experiments. Other alternatives are under study.

It can be demonstrated that the tone of color, in which an object point appears in the image, essentially depends on the axial chromatic aberration only and the transmissions of the chromatic filters. The spectral emission of the light source, the spectral transmission of the objective, the spectral sensitivity of the camera, and specifically the spectral reflectivity of the object have no influence on the tone of color. Only the intensity in which a point appears depends on the color of the object and can be zero in a disadvantageous case, in which the spectral reflectivity of the object has a narrow bandwidth.

For the determination of height by the chromatic method, a color image has to be taken from the scene by use of the three selected chromatic filters, for instance, and a black-and-white film. For each image point the color $S=\left(S_{1}, S_{2}, S_{3}\right)$ and the tone of color $\left(X_{f}, Y_{f}\right)$ must be determined. The height evaluation of the object points occurs by the appropriate evaluation of the color tone after an adequate calibration of the imaging system has taken place. For this a reference surface (plane mirror) is placed as an object into the system perpendicular to the optical axis. The mirror is shifted stepwise in the $z$ direction. Depending on the $z$ position of the mirror, the image will have different tones of color. Every tone of color indicates a certain height of the measured surface part. After that the tone of color belonging to each height is known. When measuring an object, we must, after determining the tone of color of each point, assign a height to that tone by using the calibration curve conversely. This assignment must be unequivocal, i.e., each height must have another tone of color in the calibration curve. This has been achieved by the choice of adequate chromatic filters. Figures 5-7 show the spectral transmissions of these chromatic filters, and Fig. 8 shows the measured curves of $S_{i}(z)$, which are the color components over an axial range of $5.1 \mu \mathrm{m}$. The tone of color triangle (Fig. 9) shows two dimensionally the tones of color that occur. This curve does not intersect anywhere. Therefore an unequivocal assignment of heights to tones of color is possible. 


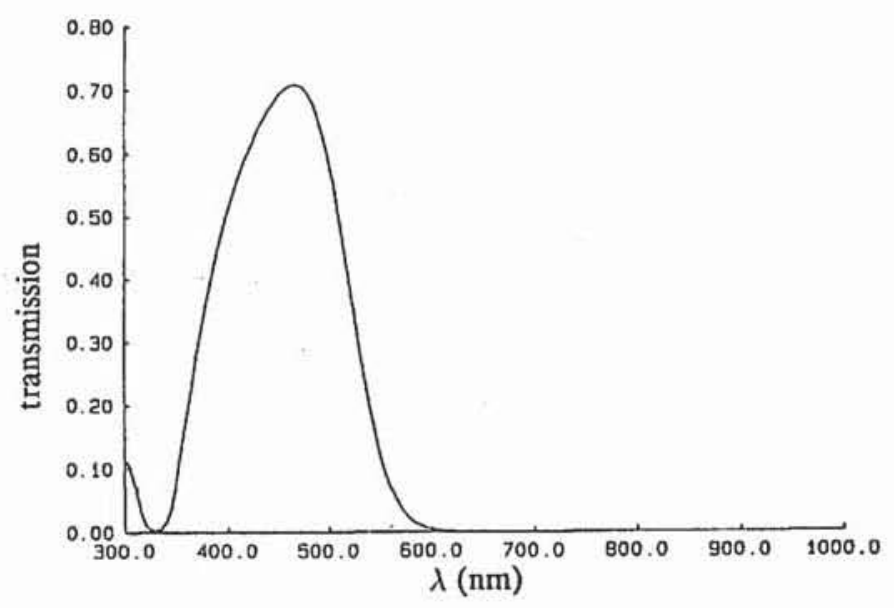

Fig. 5. Spectral transmission of the selected blue filter.

Figure 10 shows two examples for height determination on microchips with the chromatic method. For a discussion of the resolution of this method, the following considerations must be taken into account. At first it is important to know how accurately the tone of color in the image of an object point can be determined and reproduced. This depends strongly on the measurements of the intensities $S_{1}, S_{2}$, and $S_{3}$. For instance, if the noise of the camera has a relative

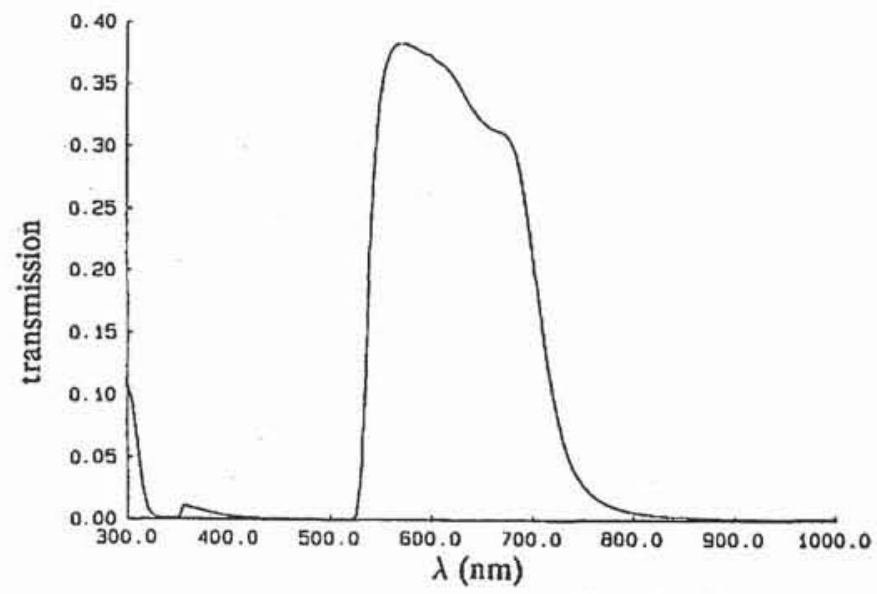

Fig. 6. Spectral transmission of the selected yellow filter.

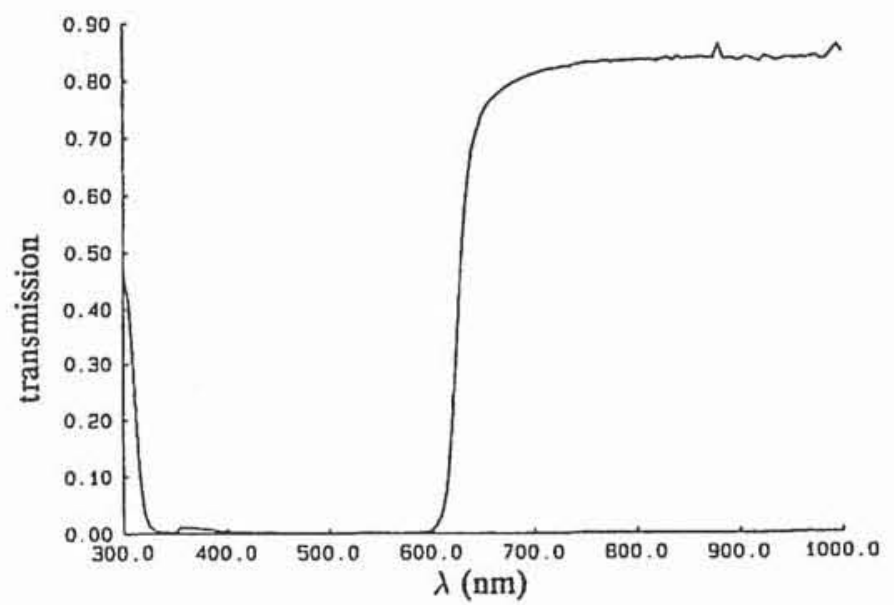

Fig. 7. Spectral transmission of the selected red filter.

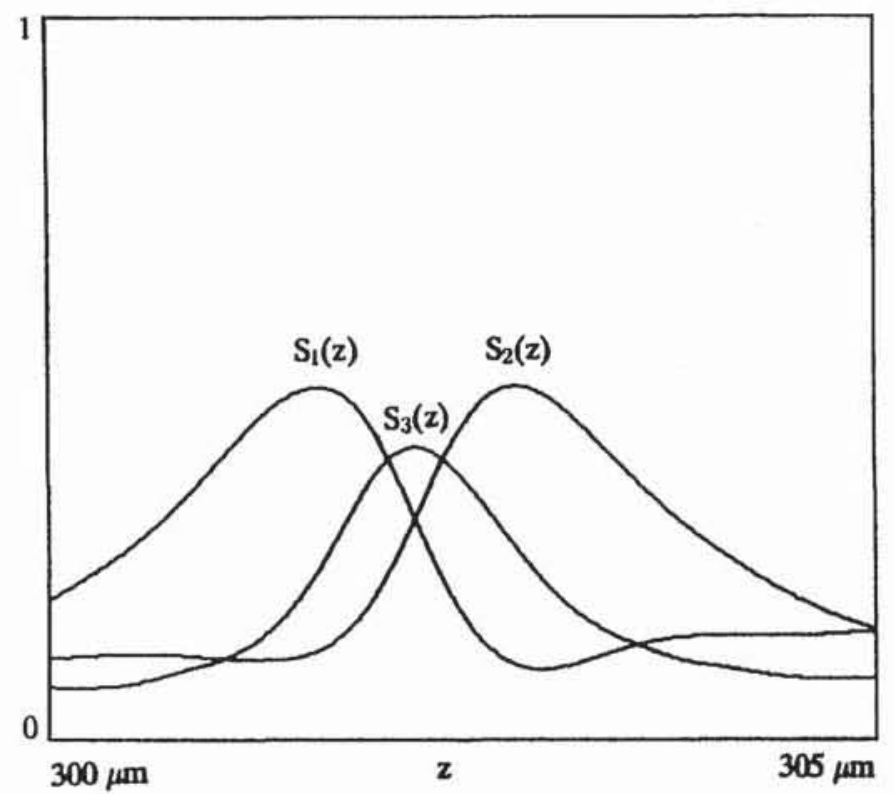

Fig. 8. Measured dependence of the color components $S_{i}$ on the axial shift $z$ by using the three selected filters.

standard deviation of $\sigma_{r}$, we obtain intensities in the range $S_{1}\left(1 \pm \sigma_{r}\right), S_{2}\left(1 \pm \sigma_{r}\right)$, and $S_{3}\left(1 \pm \sigma_{r}\right)$.

The resulting component of the tone of color $X_{f}$ is then in the range of

$$
X_{f}=\frac{S_{1}\left(1 \pm \sigma_{r}\right)}{S_{1}\left(1 \pm \sigma_{r}\right)+S_{2}\left(1 \pm \sigma_{r}\right)+S_{3}\left(1 \pm \sigma_{r}\right)} .
$$

The limits of this range are

$$
\begin{aligned}
& X_{f, \text { max }}=\frac{S_{1}\left(1+\sigma_{r}\right)}{S_{1}\left(1+\sigma_{r}\right)+S_{2}\left(1-\sigma_{r}\right)+S_{3}\left(1-\sigma_{r}\right)}, \\
& X_{f, \min }=\frac{S_{1}\left(1-\sigma_{r}\right)}{S_{1}\left(1-\sigma_{r}\right)+S_{2}\left(1+\sigma_{r}\right)+S_{3}\left(1+\sigma_{r}\right)} .
\end{aligned}
$$

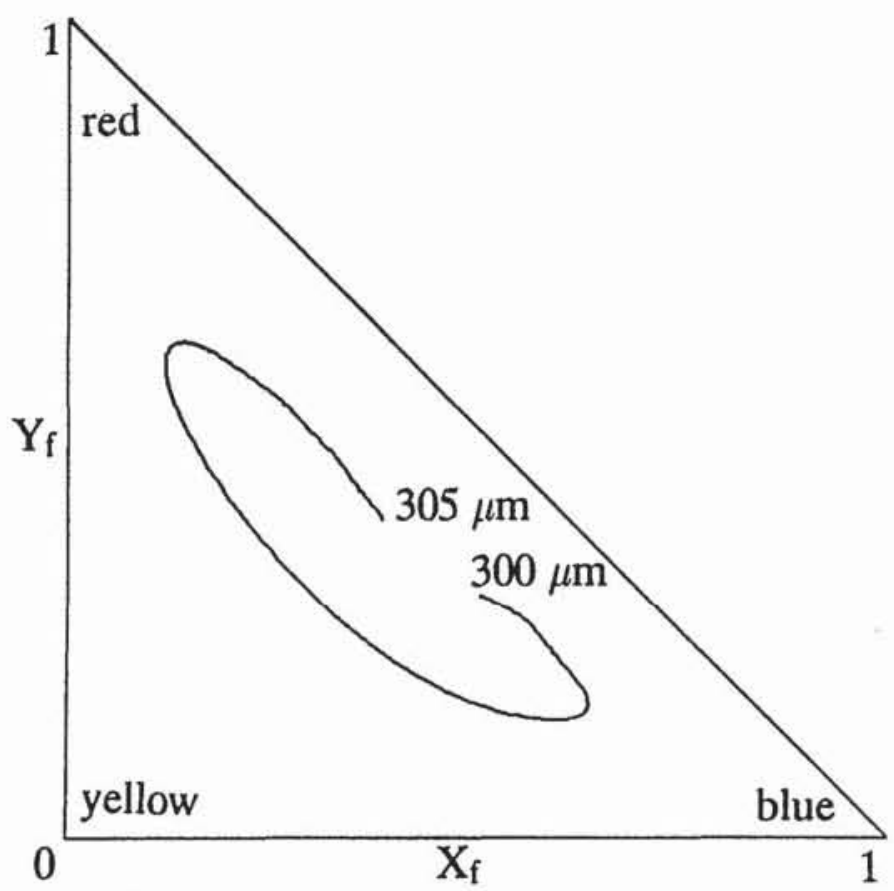

Fig. 9. Curve of tones of color by using the three selected filters. 


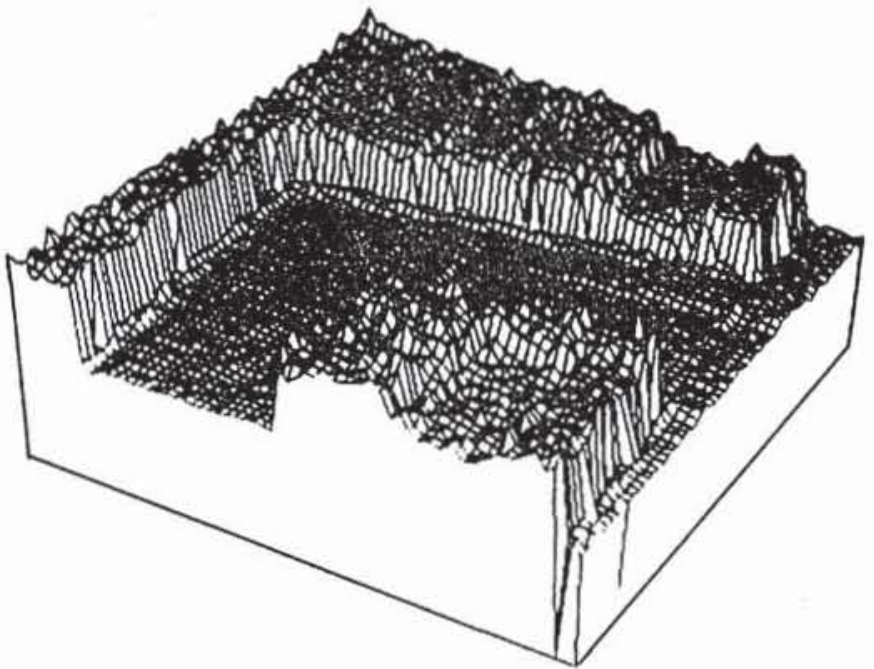

(a)

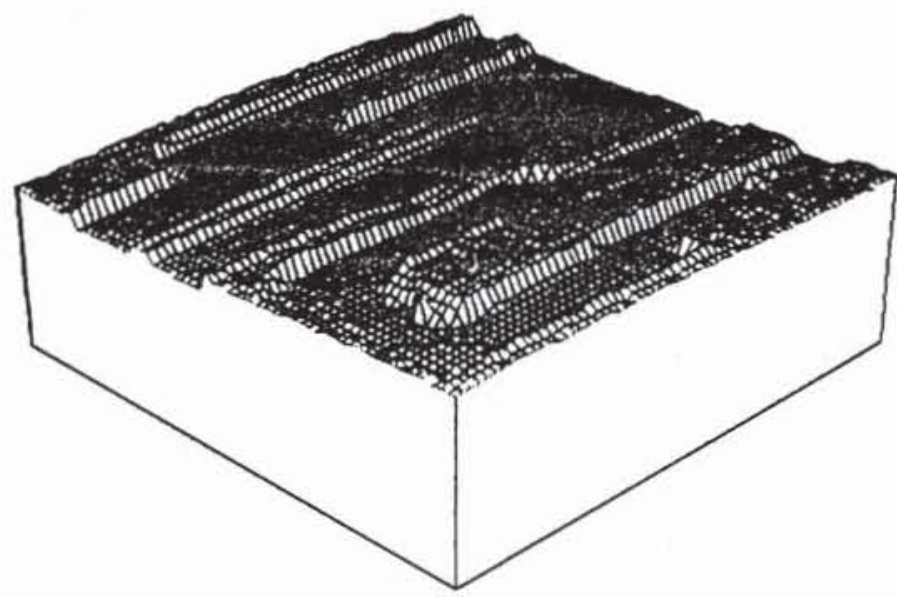

(b)

Fig. 10. Topography of two microchips measured with the chromatic method.

The extension of this range is

$$
\begin{aligned}
\Delta X_{f} & =X_{f, \max }-X_{f, \min } \\
& =\frac{4 \sigma_{r}}{2\left(1+\sigma_{r}^{2}\right)+\left(1-\sigma_{r}^{2}\right)\left[\left(S_{1} / S_{2}+S_{3}\right)+\left(S_{2}+S_{3} / S_{1}\right)\right]} \\
& \leq \sigma_{r},
\end{aligned}
$$

because $\left[\left(S_{1} / S_{2}+S_{3}\right)+\left(S_{2}+S_{3} / S_{1}\right)\right] \geq 2$ for any values of $S_{1}, S_{2}$, and $S_{3}$.

Therefore the absolute accuracy of the dimensionless tone of color $X_{f}$ is $\sigma_{r}$ or $\pm \sigma_{r} / 2$ and corresponds to half of the relative accuracy of the intensity measured by the camera. Because similar considerations hold for $Y_{f}$, the range of resolution can be assumed to be a circle within the tone of color triangle centered about the measured tone of color and having a radius of $\sigma_{r} / 2$. The tone of color triangle has an extension in the $X_{f}$ and $Y_{f}$ directions of 1 . The relative standard deviation of the camera signal $\sigma_{r}$ is $1 \%$, so the radius of the resolution circle is 0.005 . Within this circle the calibration curve passes on the average through a height range of $35 \mathrm{~nm}$. Additionally, the resolution is slightly reduced by the inaccuracy caused by discrete calibration points. Therefore an expected standard deviation for the height determination is $45 \mathrm{~nm}$. This value has been confirmed by experiments.

The advantage of the chromatic method in comparison with the height section method is that only three color images have to be taken instead of 256 height sections. Because the time needed for the entire measurement is determined mainly by the time needed for taking the images, an obvious reduction of consumed time has been achieved. This is especially interesting in the measurements of moving objects. However, the depth resolution is reduced. For larger object fields a $20 \times / 0.45$ objective was also used.

\section{Conclusion}

It has been shown that confocal microscopy is appropriate for the determination of topography with high lateral and axial resolution. By parallel processing, especially with the chromatic method, interesting perspectives for topometry have been proposed and will be analyzed further in the future.

We thank the Deutsche Forschungsgemeinschaft for financial support. We thank the reviewer for constructive comments.

\section{References}

1. T. Wilson and C. J. R. Sheppard, Theory and Practice of Scanning Optical Microscopy (Academic, London, 1984).

2. C. J. Brakenhoff, P. Blom, and P. Barends, "Confocal scanning light microscopy with high aperture immersion lenses," J. Microsc. 117, 219-232 (1979).

3. K. Carlsson and N. Aslund, "Confocal imaging for 3-D digital microscopy," Appl. Opt. 26, 3232-3238 (1987).

4. D. A. Agard and J. W. Sedat, "Three-dimensional architecture of a polytene nucleus," Nature (London) 302, 676-681 (1983).

5. A. Erhardt, G. Zinser, D. Komitowski, and J. Bille, "Reconstructing 3-D light microscopic images by digital image processing," Appl. Opt. 24, 194-200 (1985).

6. G. Q. Xiao, T. R. Corle, and G. S. Kino, "Real time confocal scanning optical microscope," Appl. Phys. Lett. 53, 716-718 (1988).

7. G. S. Kino and G. Q. Xiao, "Real time scanning optical microscopes," in Confocal Microscopy, T. Wilson, ed. (Academic, London, 1990), pp. 361-387.

8. C. J. R. Sheppard and H. J. Matthews, "The extended-focus, auto-focus and surface-profiling techniques of confocal microscopy," J. Mod. Opt. 35, 145-154 (1988).

9. N. S. Levine, "Three dimensional image visualisation using the real-time confocal scanning optical microscope," in Integrated Circuit Metrology, Inspection, and Process Control IV, W. H. Arnold, ed., Proc. Soc. Photo-Opt. Instrum. Eng. 1261, 91-101 (1990). 